\title{
Investigations of wireless transmission model based on delta function in indoor environment
}

\author{
Beata Krupanek` and Ryszard Bogacz \\ Silesian University of Technology, Faculty of Electrical Engineering Institute of Measurement Science, Electronics and Control, \\ Gliwice, Poland
}

Received: 23 December 2015 / Accepted: 6 March 2016

\begin{abstract}
The paper presents an application of probabilistic model to describe a radio propagation delay in indoor environment. Model is based of delta functions sequence to describe retransmissions between a transmitter and a receiver. Theoretical considerations have been verified by measurement results obtained by using the experimental system developed for investigating delays caused by external disturbances influencing the wireless transmission. One of such a commonly occurring disturbances are walls in building where the radio signal is propagating.
\end{abstract}

Keywords: Wireless, model, indoor, propagation, ZigBee

\section{Introduction}

In recent years, one can observe a common usage of wireless networks in measurement and control systems which causes that determination of quality of services provided by such networks becomes a crucial problem [1]. In the modern systems, the knowledge of parameters characterizing transmission channel quality allows to adequate designing the system components. One of the essential quality parameters of data transmission in such systems concerns to delays introduced by different elements of the network. The delays in networks are dependent on many factors [2], the influence of which can be described both in a deterministic and a probabilistic way. The deterministic dependencies are mainly used to determine maximum values of delays [3], whereas probabilistic means are used for determining distributions of the delays occurring as results of factors described randomly [2]. The probabilistic description of delays provides full information on their features [4,5], therefore, its introduction into the analysis of network characteristics can have a broader meaning than application of deterministic descriptions.

Nowadays, to describe communication delays in probabilistic categories, one can use different mathematical means such as queuing theory [6], Petri's nets [7] and the theory of queues applying Markov stochastic processes [8]. This paper proposes a method of probabilistic description of delays mainly directed on wireless networks and based on using the delta function [2] for description of both constant delays introduced by network devices and communication delays appearing when

^ Correspondence: beata.krupanek@polsl.pl retransmissions are performed. This model was described in details in reference [10].

Most of nowadays radio measuring systems and transmissions systems such a home automation, wireless meter reading and other operate in indoor environment. Buildings and other structures cause difficult problems for wireless (radio) communications. Whether communication is via ZigBee, Wi-Fi or personal cellular phone, communications to, from, and within structures can degrade depending on a variety of factors $[11,12]$. These factors include multipath effects, reflection from coated exterior glass, non-line-of-sight path loss, and signal absorption in the building construction materials, among others. The communications problems may be compounded by lack of a repeater to amplify and retransmit the signal or by poor placement of the repeater. RF propagation in structures can be so poor that there may be areas where the signal is virtually nonexistent, rendering radio communication impossible [11]. The paper show a description and measurements of delays in different indoor structures.

\section{Brief characteristics of the model}

It has been assumed that all devices in the network use the same transmission medium, therefore, to send data trough a communication channel, the active transmitters have to compete each other in order to get access to the medium. The access procedure is of random character [9], moreover, the data transmission trough the medium is disturbed by different kinds of random factors $[2,9]$. In this physical conditions, the total delay of data transmission in every 


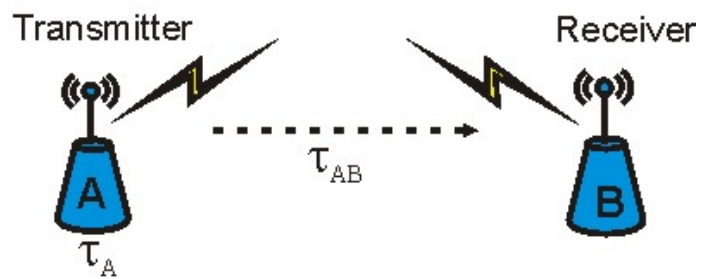

Fig. 1. Sources of delays during transmission from node A to $\mathrm{B}, \tau_{\mathrm{A}}-$ time necessary to obtain the access to a communication medium, $\tau_{\mathrm{AB}}$ - time of the message transmission.

communication chain created in the network is of random character, too.

The essence of the method of modelling delays in wireless networks presented in the paper consists in using delta function series which are treated as mathematical descriptions of probability density functions of delays in communications chains. The partial model describing a delay of a transmission between two devices is relatively simple in this case and its important feature is the possibility of containing influence of disturbances on this delay. The results of investigations of such an influence for different kinds of disturbances, passive and active, have been presented in reference [2].

\subsection{General model of delays}

The simple situation is shown in Figure 1, where A and B denote nodes of a wireless network which communicate directly.

A model of the total delay has to contain all possible values of the delays occurring in the described conditions [10]. If one takes into account that access procedures commonly used in wireless networks are of random character [1], the total delay should be described in probabilistic categories. Such a description can be obtained by using the Dirac's delta [6] as a probability density function. The convolution of the probability density function $g(\tau)$ with the delta function gives [10]:

$$
g(\tau) \otimes \delta(\tau-b)=g(\tau-b),
$$

which means that this convolution shifts the function $g(\tau)$ in the horizontal axis by the value $b$. It allows simplifying transformations by making such displacements instead of performing the convolutions. Basing on properties on delta function [6] the probability density function of the access delay can be write in the form:

$$
g_{\mathrm{A}}\left(\tau_{\mathrm{A}}\right)=g_{\text {Apat }}\left(\tau_{\mathrm{A}}-\tau_{0}\right),
$$

where $\tau_{0}$ is the expected value of $g_{\mathrm{A}}\left(\tau_{\mathrm{A}}\right)$.

This function plays an important role in when it is used as a kind of a pattern in the delay model built on the basis of measurements. The results enable obtaining a pattern for ZigBee module as the normal probability density function $g_{\text {Apat }}\left(\tau_{\mathrm{A}}\right)=N(0,1)$ ms properly truncated because $g_{\mathrm{A}}\left(\tau_{\mathrm{A}}\right)>0$.

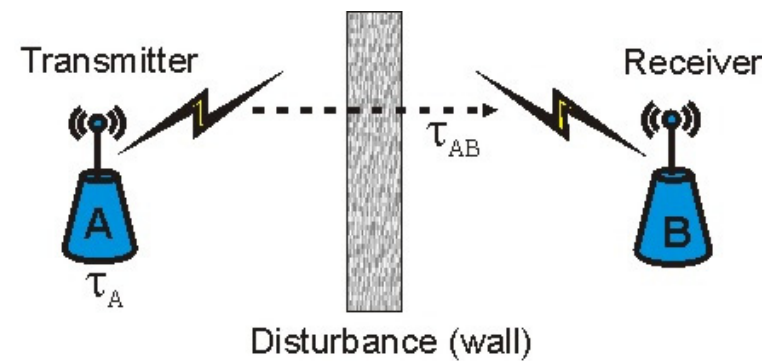

Fig. 2. Transmission between two nodes disturbed by a wall.

\subsection{Model of delays for indoor environment}

The radio signal being the communication medium in wireless networks is exposed to many different disturbances both of passive and active nature $[4,10]$. The passive disturbances are caused by terrain obstacles, walls, etc. while the active ones result from influences of electromagnetic and electrostatic fields.

Generally, the appearance of disturbances cause that the received message is not correct. Information about this fact is sent back to the transmitter which usually tries to send the message once more. The number of retransmissions depends on construction of the wireless module and can be determined by its user.

In Figure 2 there is shown the situation when two nodes $\mathrm{A}$ and $\mathrm{B}$ communicate directly but a disturbance, represented in this figure by a wall, causes that some transmissions have to be repeated, i.e. the message must be retransmitted. It means that the partial delay $\tau_{\mathrm{AB}}$ connected with the correct transmission of a message is not constant as it has been considered assumed previously but depends on the number of retransmissions. In the work [2], it has been experimentally proved that in this case $\tau_{\mathrm{AB}}$ can be represented by the following delta function sequence:

$$
\begin{aligned}
g_{\mathrm{AB}}\left(\tau_{\mathrm{AB}}\right)= & a_{0} \delta\left(\tau_{\mathrm{AB}}-b_{0}\right)++a_{1} \delta\left(\tau_{\mathrm{AB}}-b_{1}\right)+\ldots \\
& +a_{k} \delta\left(\tau_{\mathrm{AB}}-b_{k}\right),
\end{aligned}
$$

where $\delta($.$) denotes the delta function, k$ is the number of retransmissions. Both $a_{0}, a_{1}, \ldots, a_{k}$ and $b_{0}, b_{1}, \ldots, b_{k}$ are constant coefficients with non-negative values.

The total communication delay $\tau_{\text {tot }}$, can be described by equation resulting from (1) and (3) what was shown in reference [10]:

$$
\begin{aligned}
g_{\text {tot }}\left(\tau_{\text {tot }}\right)= & a_{0} g_{\mathrm{A}}\left(\tau_{\text {tot }}-b_{0}\right)++a_{1} g_{\mathrm{A}}\left(\tau_{\text {tot }}-b_{1}\right)+\ldots \\
& +a_{k} g_{\mathrm{A}}\left(\tau_{\text {tot }}-b_{k}\right)
\end{aligned}
$$

which is the sum of duplicates of the probability density function $g_{\mathrm{A}}(\cdot)$ properly moved in time by the constant values $b_{0}, b_{1}, \ldots, b_{k}$ and multiplied by the constant coefficients $a_{0}, a_{1}, \ldots, a_{k}$, which describe the probability of occurring the succeeding retransmissions.

Equation (4) is the probabilistic model of the total communication delay in indoor environment when disturbances affect the transmission, which causes the necessity 


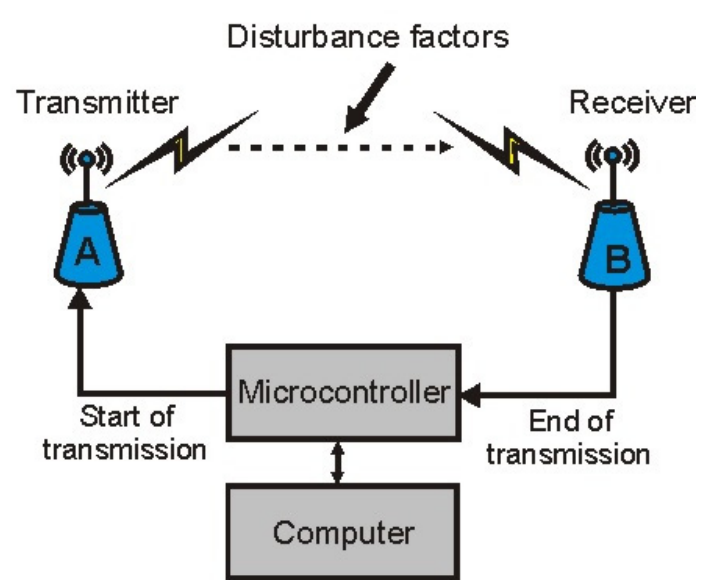

Fig. 3. Scheme of the system for measurements of transmission delays.

of retransmissions. Instead of using the function $g_{\mathrm{A}}(\cdot)$ in this model, it is better to introduce the pattern $g_{\text {Apat }}(\cdot)$ described by (2), which has the expected value equal to 0 . In this case the time displacements $b_{0}, b_{1}, \ldots, b_{k}$ can be determined in relation to the vertical axis as constant delays $\tau_{0}, \tau_{1}, \ldots, \tau_{k}$, which allows calculating them on the basis of measurement results. Taking this into account, on can write the parameters of the model describing the delays of the disturbed communication on the way between nodes $\mathrm{A}$ and $\mathrm{B}$ as:

$$
\operatorname{Delay}_{\mathrm{AB}}\left\{g_{\text {Apat }}(.),\left(a_{0}, \tau_{0}\right),\left(a_{1}, \tau_{1}\right), \ldots,\left(a_{k}, \tau_{k}\right)\right\} \text {. }
$$

To verify the thesis that this model is a good description of delays in wireless networks exposed to disturbances, the total delays in real networks were measured with use of the developed system and parameters of the model were identified for several kinds of external disturbances.

\section{Measurement system}

The main practical application of the proposed mathematical apparatus consists in description of communication delays by the model, parameters of which depend on properties of factors disturbing the transmission medium. To identify the model, it is necessary to measure delays in selected conditions. The general scheme of the system used for measurements of communication delays in wireless networks is shown in Figure 3.

The investigated communication channel consists of two elements: a transmitter and a receiver working in ZigBee standard $[3,9]$. Then used radio modules were Xbee's from Digi International. Transmission of data having a constant length is disturbed by different kind of factors such as walls, electromagnetic or electrostatic fields and other wireless transmissions. The delay, defined as the time between the start of sending data in the transmitter and the moment when data are completed in the receiver, is measured by a microcontroller.

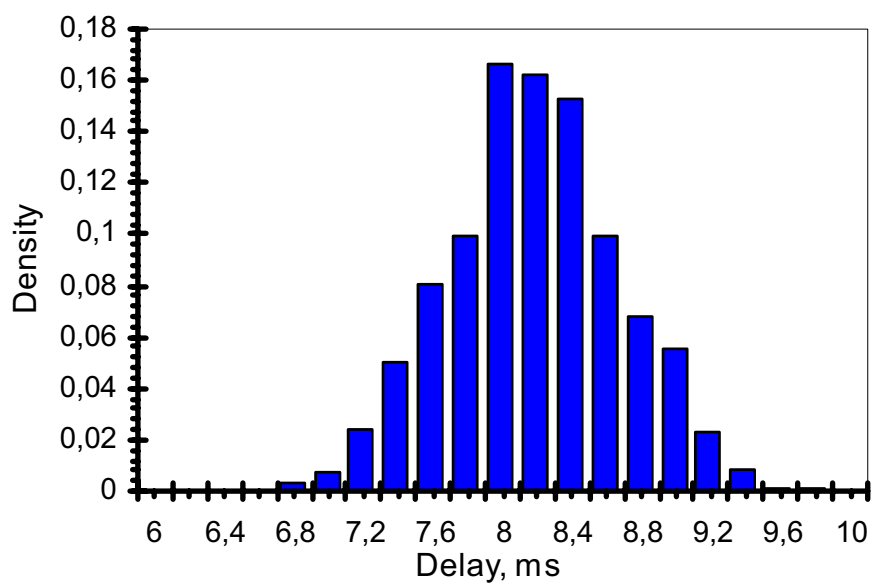

Fig. 4. Probability density function pattern.

Table 1. RSSI ratio measurement results.

\begin{tabular}{cc}
\hline Kind of wall & RSSI ratio, dBm \\
\hline 1 & -22 \\
2 & -28 \\
3 & -33 \\
4 & -41 \\
5 & -35 \\
6 & -54 \\
\hline
\end{tabular}

\section{Experimental results}

The tests were conducted in indoor environment for the various walls of different construction. During the measurements the ratio RSSI and transmission delay between wireless nodes were registered. In order to obtain a probability density function pattern measurements were taken in an environment without passive and active disturbances. The result is shown in Figure 4.

The model for histogram from Figure 4 is given by equation:

$$
g_{\text {tot }}\left(\tau_{\text {tot }}\right)=1 \cdot g_{\mathrm{A}}\left(\tau_{\text {tot }}-8.2\right) .
$$

The horizontal shift is $8.20 \mathrm{~ms}$ and all transmitted by A node data packets were received by node B. This histogram is called a pattern because it describes a delay without any passive or active disturbance.

Then, measurements were performed for disturbances factors like:

1. Single brick wall $150 \mathrm{~mm}$ thick.

2. Single brick wall $300 \mathrm{~mm}$ thick.

3. Double brick wall $150 \mathrm{~mm}$ thick each.

4. Double brick wall $300 \mathrm{~mm}$ thick each.

5. Single concrete wall $100 \mathrm{~mm}$ thick.

6. Double concrete wall $100 \mathrm{~mm}$ thick each.

Table 1 shows the results of measuring the RSSI ratio. The results may differ in similar indoor situations because of specific properties of used materials and such a parameters like temperature and humidity. 


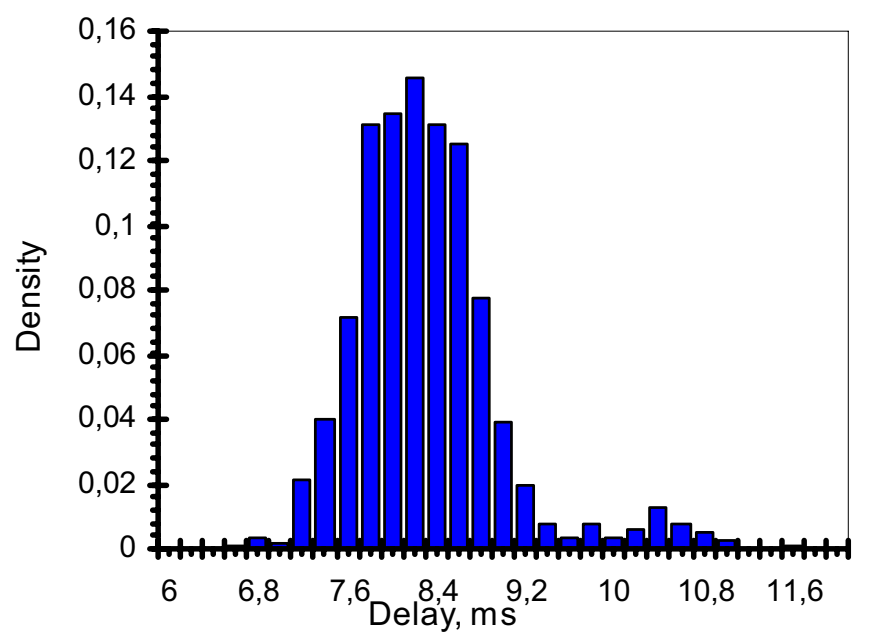

Fig. 5. Delay histogram for single brick wall, $150 \mathrm{~mm}$ thick.

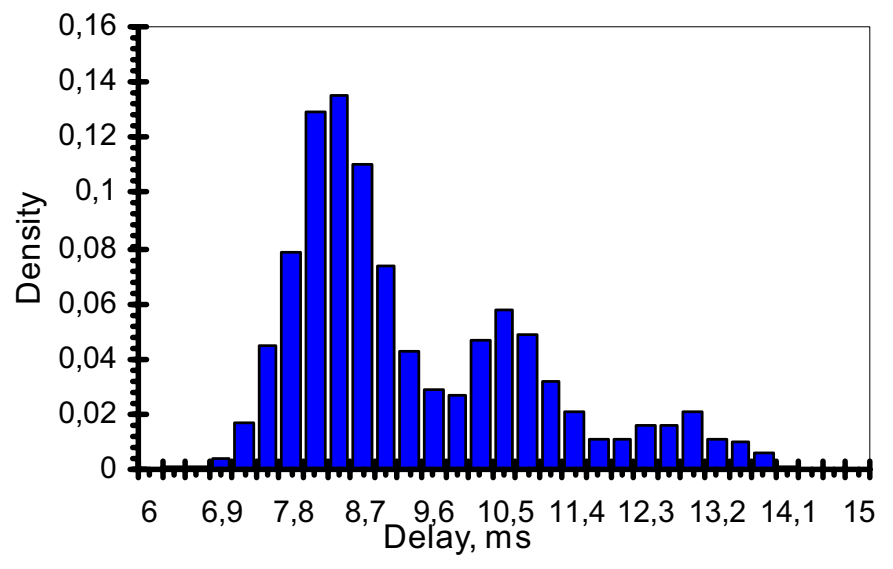

Fig. 6. Delay histogram for single brick wall, $300 \mathrm{~mm}$ thick.

From the table above appears that concrete attenuates the radio signal much more then bricks. A radio signal passing through the two thin walls is more attenuated than passing through a single wall with a thickness being the sum of single wall thickness. This is due to the phenomenon of attenuation, scattering and multipath [11]. This parameters measurements was also studied in [12].

Figures from 5 to 7 shows the example results of communication delay measurement for 1, 2 and 5 (from Tab. 1) situations respectively.

Figure 5 presents situation when not all of transmitted packets reach the receiver by the first time. Five percent of them were retransmitted with average delay $10.4 \mathrm{~ms}$. The obtained model from situation described on Figure 5 can be written as:

$$
g_{\text {tot }}\left(\tau_{\text {tot }}\right)=0.95 g_{\mathrm{A}}\left(\tau_{\text {tot }}-8.2\right)+0.05 g_{\mathrm{A}}\left(\tau_{\text {tot }}-10.4\right)
$$

In situation shown in Figure 6 we have three pattern distributions shifted by $8.2 \mathrm{~ms}, 10.4 \mathrm{~ms}$ and $12.6 \mathrm{~ms}$. That means the radio signal is highly attenuated and part of

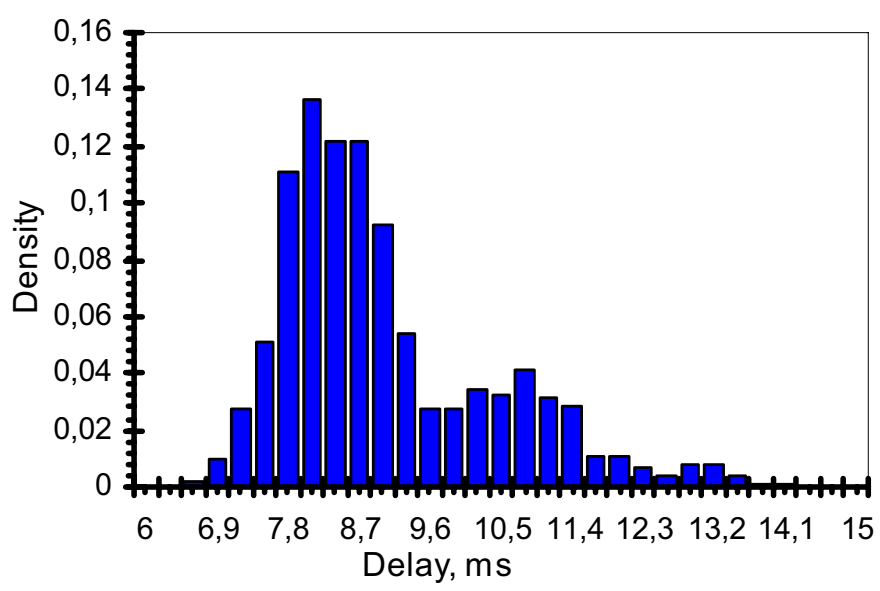

Fig. 7. Delay histogram for single concrete wall, $100 \mathrm{~mm}$ thick.

packets $(9 \%)$ were retransmitted second time (8):

$$
\begin{aligned}
g_{\text {tot }}\left(\tau_{\text {tot }}\right)= & 0.65 g_{\mathrm{A}}\left(\tau_{\text {tot }}-8.2\right)+0.26 g_{\mathrm{A}}\left(\tau_{\text {tot }}-10.4\right) \\
& +0.09 g_{\mathrm{A}}\left(\tau_{\text {tot }}-12.6\right) .
\end{aligned}
$$

For transmission by one concrete wall radio signal is also highly attenuated but only $4 \%$ of transmitted packets are retransmitted second time. It shows that one concrete wall less affected the communication delay then two brick walls.

$$
\begin{aligned}
g_{\text {tot }}\left(\tau_{\text {tot }}\right)= & 0.74 g_{\mathrm{A}}\left(\tau_{\text {tot }}-8.2\right)+0.22 g_{\mathrm{A}}\left(\tau_{\text {tot }}-10.4\right) \\
& +0.04 g_{\mathrm{A}}\left(\tau_{\text {tot }}-12.6\right)
\end{aligned}
$$

Other performed measurements shows that combination of concrete and brick walls affected transmission delays so much that part of packets can be lost.

\section{Conclusions}

The presented model allows for the description of the communication delays taking into account the data retransmission caused by passive disturbances such as walls and partitions in the environment inside the building. The model is also very easy to implementation in simulation environment like OPNET Modeler $[2,3]$. Other performed measurements shows that combination of concrete and brick walls affected transmission delays so much that part of packets can be lost.

\section{References}

1. L.F. Akyildiz, W. Su, Y. Sankarasubramanian, E. Cayirci: A survey on sensor networks, IEEE Commun. Mag. 40, 102-114 (2002)

2. B. Krupanek, Modeling of transmission delays caused by disturbances in the wireless networks in IEEE 802.15.4 standard (in Polish), Ph.D. thesis, Gliwice, 2012 
3. T. Topór-Kamiński, B. Krupanek, J. Homa, Delays models of measurement and control data transmission network, in Advanced Technologies for Intelligent Systems of National Border Security, Studies in Computational Intelligence, 440 (pp. 257-279)

4. J. Jakubiec, Errors and uncertainties in measurement and control system (in Polish) (Silesian University of Technology Publishing House, Gliwice, 2010)

5. R. Oliver, G. Fohler, Probabilistic estimation of end-toend path latency in wireless sensor networks, in IEEE Conference Mobile Adhoc and Sensor Systems, 2009, pp. $423-431$

6. P. Nain, Basic elements of queuing theory. Application to the modeling of computer systems, in Lecture Notes (University of Massachusetts, Amherst, 1998)
7. A. Bobbio, System modelling with petri nets (System Reliability Assessment, Kluwer, 1990), pp. 102-143

8. C. Grinstead, J. Snell, Introduction to probability (American Mathematical Society, 2008)

9. F. Eady, Hands-on zigbee: Implementing 802.15.4 with microcontrollers (Elsevier Inc., 2007)

10. B. Krupanek, R. Bogacz, Modelling of communication delays in wireless networks, in IMEKO TC10 Conference, Warsaw, 2014

11. Indoor Path Loss (Digi Internetional Materials, 2012)

12. K. Sahu, C. Dhanunjay, C. Sankar, Study of RF propagation losses in homogeneous brick and concrete walls using analytical frequency dependent models, IOSR J. Electron. Commun. Eng. 9 (2014) 